\title{
Selective Synthesis of 5- or 6-Aryl Octahydrocyclopenta[b]pyrroles from a Common Precursor Through Control of Competing Pathways in a Pd-Catalyzed Reaction
}

\author{
Joshua E. Ney and John P. Wolfe* \\ Department of Chemistry, University of Michigan, 930 N. University Avenue, Ann Arbor, \\ Michigan 48109-1055
}

\section{Supporting Information}

Experimental procedures and characterization data for new compounds in equations 3-4 and Tables 4-5, complete descriptions of stereochemical assignments, and descriptions of optimization studies (21 pages).

General All reactions were carried out under an argon atmosphere in flame-dried glassware. Tris(dibenzylidineacetone)dipalladium $(0)$ and all phosphine ligands except those noted below were purchased from Strem Chemical Co. and used without further purification. All aryl bromides except for 4-bromobenzoic acid tert-butyl ester were obtained from commercial sources (Aldrich Chemical Co. or Acros Chemical Co.) and were used as obtained. 4-Bromobenzoic acid tert-butyl ester $^{1}, \quad(2-c y c l o p e n t-2-e n y l e t h y l)-(4-m e t h o x y p h e n y l) a m i n e \quad(1 \mathbf{a})^{2}, \quad 1,2-b i s[\operatorname{bis}(p-$ trifluoromethylphenyl)phosphino] ethane ${ }^{3}, 1,2-$ bis(di- $p$-methoxyphenylphosphino)ethane ${ }^{3}, \operatorname{tris}(o-$ trifluoromethylphenyl)phosphine ${ }^{4}, \quad \operatorname{tr}$ is ( $m$-trifluoromethylphenyl)phosphine ${ }^{4}, \quad \operatorname{tr}$ is $(p$ trifluoromethylphenyl)phosphine ${ }^{4}$, and tri( $p$-methoxyphenyl)phosphine ${ }^{5}$ were prepared according to literature procedures. Toluene was purified using a GlassContour solvent purification system. 
Yields refer to isolated yields of compounds estimated to be $\geq 95 \%$ pure as determined by ${ }^{1} \mathrm{H}$ NMR and/or combustion analysis. The yields reported in the supporting information describe the result of a single experiment, whereas the yields reported in equations 1 and 2 and Tables 1 and 2 are average yields of two or more experiments. Thus, the yields reported in the supporting information may differ from those shown in equations 1 and 2 and Tables 1 and 2.

\section{Synthesis of $\mathrm{N}$-(2-Cyclopent-2-enylethyl)arylamines (1a-e)}

$N$-(2-Cyclopent-2-enylethyl)-4-chloroaniline (1b). A flame-dried round-bottom flask was charged with 1,1'-carbonyl diimidazole (2.7 g, $16.6 \mathrm{mmol})$, purged with argon, and then THF (20 mL) and 2-cyclopenten-1-acetic acid (2 mL, $2.09 \mathrm{~g}, 16.6 \mathrm{mmol})$ were added via syringe. The mixture was stirred at room temperature for $1.5 \mathrm{~h}$ and then 4-chloroaniline $(2.1 \mathrm{~g}, 16.6 \mathrm{mmol})$ was added via syringe and the resulting mixture was stirred at room temperature for $3.5 \mathrm{~h}$. The reaction mixture was diluted with ethyl acetate $(75 \mathrm{~mL})$ and $\mathrm{H}_{2} \mathrm{O}(50 \mathrm{~mL})$, the layers were separated, and the aqueous layer was extracted with ethyl acetate $(2 \times 100 \mathrm{~mL})$. The combined organic extracts were washed with aqueous $1 \mathrm{M} \mathrm{HCl}(50 \mathrm{~mL})$ and then saturated aqueous $\mathrm{NaHCO}_{3}(50 \mathrm{~mL})$, dried over anhydrous sodium sulfate, filtered, and concentrated in vacuo to afford $3.75 \mathrm{~g}(96 \%)$ of $N$-(4-chlorophenyl)-2-(cyclopent-2-enyl)acetamide as a white solid, m.p. 130-132 ${ }^{\circ} \mathrm{C} .{ }^{1} \mathrm{H}$ NMR $\left(400 \mathrm{MHz}, \mathrm{CDCl}_{3}\right) \delta 7.47(\mathrm{~d}, J=8.8 \mathrm{~Hz}, 2 \mathrm{H}), 7.28(\mathrm{~d}, J=8.8 \mathrm{~Hz}, 2 \mathrm{H})$, $7.13(\mathrm{~s}, 1 \mathrm{H}), 5.84-5.80(\mathrm{~m}, 1 \mathrm{H}), 5.75-5.71(\mathrm{~m}, 1 \mathrm{H}), 3.26-3.16(\mathrm{~m}, 1 \mathrm{H}), 2.46-2.30(\mathrm{~m}, 3 \mathrm{H})$, $2.24-2.14(\mathrm{~m}, 1 \mathrm{H}), 1.58-1.48(\mathrm{~m}, 2 \mathrm{H})$.

A flame-dried round-bottom flask was charged with $N$-(4-chlorophenyl)-2-(cyclopent-2enyl)acetamide, purged with argon, and then THF $(16 \mathrm{~mL})$ was added via syringe. The resulting solution was cooled to $0{ }^{\circ} \mathrm{C}$ and a solution of $\mathrm{LiAlH}_{4}$ in ether $(48 \mathrm{~mL}, 48 \mathrm{mmol}, 1.0 \mathrm{M})$ was 
added dropwise via syringe. The reaction mixture was then warmed to room temperature for $21 \mathrm{~h}$ at which time the starting material had been consumed as judged by TLC analysis. The reaction mixture was cooled to $0^{\circ} \mathrm{C}$, diluted with ether $(300 \mathrm{~mL})$, and quenched slowly with aqueous 10 $\mathrm{M} \mathrm{NaOH}$ until all insoluble material had precipitated. The organic supernatant was decanted to a clean Erlenmeyer flask and the precipitate was washed with ether $(200 \mathrm{~mL})$. The combined organic extracts were diluted further with hexane $(200 \mathrm{~mL})$, dried over anhydrous sodium sulfate, filtered, and concentrated in vacuo. The crude product was then purified by flash chromatography on silica gel to afford $2.83 \mathrm{~g}(91 \%)$ of the title compound as a colorless oil. ${ }^{1} \mathrm{H}$ $\operatorname{NMR}\left(500 \mathrm{MHz}, \mathrm{CDCl}_{3}\right) \delta 7.15(\mathrm{~d}, J=9.0 \mathrm{~Hz}, 2 \mathrm{H}), 6.54(\mathrm{~d}, J=9.0 \mathrm{~Hz}, 2 \mathrm{H}), 5.83-5.80(\mathrm{~m}, 1$ H), 5.75-5.72 (m, $1 \mathrm{H}), 3.64(\mathrm{~s}, 1 \mathrm{H}), 3.18-3.09$ (m, $2 \mathrm{H}), 2.84-2.76(\mathrm{~m}, 1 \mathrm{H}), 2.47-2.31$ (m, 2 H), 2.18-2.10 (m, $1 \mathrm{H}), 1.79-1.70(\mathrm{~m}, 1 \mathrm{H}), 1.66-1.58(\mathrm{~m}, 1 \mathrm{H}), 1.56-1.45(\mathrm{~m}, 1 \mathrm{H}) ;{ }^{13} \mathrm{C}$ NMR $\left(125 \mathrm{MHz}, \mathrm{CDCl}_{3}\right) \delta 146.9,134.2,130.8,128.9,121.4,113.6,43.2,42.6,35.5,31.9,29.7$; IR (film) $3416,1601,1503 \mathrm{~cm}^{-1}$. Anal calcd for $\mathrm{C}_{13} \mathrm{H}_{16} \mathrm{ClN}$ : C, 70.42; H, 7.27; N, 6.32. Found: C, 70.58; H, 7.30; N, 6.44.

$N$-(2-Cyclopent-2-enylethyl)amine. A flame-dried round-bottomed flask was charged with 2-cyclopenten-1-acetic acid (20 mL, $21.0 \mathrm{~g}, 166 \mathrm{mmol})$ and thionyl chloride (48 mL, $79 \mathrm{~g}, 664$ mmol) and was stirred at room temperature for $2 \mathrm{~h}$. The excess thionyl chloride was removed by distillation to afford a crude acid chloride product, which was added dropwise via syringe to 28 $\%$ aqueous ammonium hydroxide $(600 \mathrm{~mL})$ with stirring. The resulting mixture was stirred at room temperature for $48 \mathrm{~h}$ then was extracted with ethyl acetate $(3 \times 250 \mathrm{~mL})$. The combined organic extracts were dried over anhydrous sodium sulfate, filtered, and concentrated in vacuo to afford $14.7 \mathrm{~g}(71 \%)$ of 2-(cyclopent-2-enyl)acetamide as a tan solid, m.p. $119-122{ }^{\circ} \mathrm{C} .{ }^{1} \mathrm{H}$ NMR 
(400 MHz, $\left.\mathrm{CDCl}_{3}\right) \delta 5.81-5.77(\mathrm{~m}, 1 \mathrm{H}), 5.73-5.68(\mathrm{~m}, 1 \mathrm{H}), 5.37(\mathrm{~s}, 2 \mathrm{H}), 3.16-3.07(\mathrm{~m}, 1 \mathrm{H})$, 2.40-2.10 (m, $5 \mathrm{H}), 1.53-1.43(\mathrm{~m}, 1 \mathrm{H})$.

A flame-dried round-bottom flask was charged with 2-(cyclopent-2-enyl)acetamide, purged with argon, and then ether $(120 \mathrm{~mL})$ was added via syringe. The resulting suspension was cooled to $0{ }^{\circ} \mathrm{C}$ and a solution of $\mathrm{LiAlH}_{4}$ in ether $(352 \mathrm{~mL}, 352 \mathrm{mmol}, 1.0 \mathrm{M})$ was added via syringe. The reaction mixture was then warmed to room temperature for $18 \mathrm{~h}$ at which time the starting material had been consumed as judged by TLC analysis. The reaction mixture was cooled to 0 ${ }^{\circ} \mathrm{C}$, diluted with ether $(1300 \mathrm{~mL})$, and quenched slowly with aqueous $10 \mathrm{M} \mathrm{NaOH}$ until all insoluble material had precipitated. The organic supernatant was decanted to a clean Erlenmeyer flask and the precipitate was washed with ether $(200 \mathrm{~mL})$. The combined organic extracts were diluted further with pentane $(500 \mathrm{~mL})$, dried over anhydrous sodium sulfate, filtered, and concentrated in vacuo. The crude product was then distilled from calcium hydride to afford 9.24 $\mathrm{g}(71 \%)$ of the title compound as a colorless liquid, b.p. $120{ }^{\circ} \mathrm{C} .{ }^{1} \mathrm{H}$ NMR $\left(400 \mathrm{MHz}, \mathrm{CDCl}_{3}\right) \delta$ 5.73-5.69 (m, $1 \mathrm{H}), 5.68-5.63(\mathrm{~m}, 1 \mathrm{H}), 2.75-2.64(\mathrm{~m}, 3 \mathrm{H}), 2.39-2.20(\mathrm{~m}, 2 \mathrm{H}), 2.08-1.98(\mathrm{~m}$, $1 \mathrm{H}), 1.61-1.50(\mathrm{~m}, 1 \mathrm{H}), 1.47-1.35(\mathrm{~m}, 2 \mathrm{H}), 1.10$ (s, br, $2 \mathrm{H}) ;{ }^{13} \mathrm{C} \mathrm{NMR}\left(100 \mathrm{MHz}, \mathrm{CDCl}_{3}\right) \delta$ 134.8, 130.4, 43.1, 40.9, 40.2, 31.9, 29.8; IR (film) 3339, 1570, $1488 \mathrm{~cm}^{-1}$.

4-(2-Cyclopent-2-enylethylamino)benzonitrile (1c). A flame-dried Schlenk flask was charged with $\mathrm{Pd}_{2}(\mathrm{dba})_{3}(110 \mathrm{mg}, 0.12 \mathrm{mmol}, 1 \mathrm{~mol} \%)$, 2-(di-tert-butylphosphino)biphenyl (10) (72 mg, $0.24 \mathrm{mmol}, 2 \mathrm{~mol} \%), \mathrm{NaOt}$-Bu (2.81 g, $28.8 \mathrm{mmol})$, and 4-bromobenzonitrile (2.2 g, $12.0 \mathrm{mmol})$. The flask was purged with argon, and toluene $(50 \mathrm{~mL})$ was added followed by $N-(2-$ cyclopent-2-enylethyl)amine $(2.0 \mathrm{~g}, 18.0 \mathrm{mmol})$ via syringe. The resulting mixture was heated to $100{ }^{\circ} \mathrm{C}$ with stirring until the starting material had been consumed as judged by GC analysis (1.5 h). The reaction mixture was cooled to room temperature, quenched with saturated aqueous 
ammonium chloride $(50 \mathrm{~mL})$ and diluted with ethyl acetate $(100 \mathrm{~mL})$. The layers were separated and the aqueous layer was extracted with ethyl acetate $(2 \times 100 \mathrm{~mL})$. The combined organic extracts were dried over anhydrous sodium sulfate, filtered, and concentrated in vacuo. The crude product was then purified by flash chromatography on silica gel to afford $1.79 \mathrm{~g}(72 \%)$ of the title compound as a yellow solid, m.p. $44-45{ }^{\circ} \mathrm{C} .{ }^{1} \mathrm{H}$ NMR $\left(400 \mathrm{MHz}, \mathrm{CDCl}_{3}\right) \delta 7.36(\mathrm{~d}, J=$ $8.8 \mathrm{~Hz}, 2 \mathrm{H}), 6.53(\mathrm{~d}, J=8.8 \mathrm{~Hz}, 2 \mathrm{H}), 5.76-5.72(\mathrm{~m}, 1 \mathrm{H}), 5.68-5.64(\mathrm{~m}, 1 \mathrm{H}), 4.49$ (br s, $1 \mathrm{H})$, 3.22-3.08 (m, 2 H), 2.79-2.70 (m, 1 H), 2.41-2.22 (m, 2 H), 2.13-2.02 (m, 1 H), 1.77-1.67 (m, $1 \mathrm{H}), 1.65-1.54(\mathrm{~m}, 1 \mathrm{H}), 1.48-1.38(\mathrm{~m}, 1 \mathrm{H}) ;{ }^{13} \mathrm{C} \mathrm{NMR}\left(100 \mathrm{MHz}, \mathrm{CDCl}_{3}\right) \delta 151.4,133.7$, 133.3, 130.9, 120.4, 111.8, 97.6, 42.9, 41.5, 34.9, 31.7, 29.5; IR (film) 3370, 2212, 1608, 1526 $\mathrm{cm}^{-1}$. Anal calcd for $\mathrm{C}_{14} \mathrm{H}_{16} \mathrm{~N}_{2}: \mathrm{C}, 79.21 ; \mathrm{H}, 7.60 ; \mathrm{N}, 13.20$. Found: $\mathrm{C}, 79.15 ; \mathrm{H}, 7.60 ; \mathrm{N}, 13.03$.

4-(2-Cyclopent-2-enylethylamino)benzoic acid tert-butyl ester (1d). A flame-dried Schlenk flask was charged with $\mathrm{Pd}_{2}(\mathrm{dba})_{3}(110 \mathrm{mg}, 0.12 \mathrm{mmol}, 1 \mathrm{~mol} \%)$, 2-(di-tertbutylphosphino)biphenyl (10) (72 mg, $0.24 \mathrm{mmol} 2 \mathrm{~mol} \%$ ), and $\mathrm{NaO} t-\mathrm{Bu}(2.81 \mathrm{~g}, 28.8 \mathrm{mmol})$. The flask was purged with argon, and toluene $(50 \mathrm{~mL})$ was added followed by $N$-(2-cyclopent-2enylethyl)amine (2.0 g, $18.0 \mathrm{mmol})$ and 4-bromobenzoic acid tert-butyl ester $(3.1 \mathrm{~g}, 12.0 \mathrm{mmol})$ via syringe. The resulting mixture was heated to $100{ }^{\circ} \mathrm{C}$ with stirring until the starting material had been consumed as judged by GC analysis $(3 \mathrm{~h})$. The reaction mixture was cooled to room temperature, quenched with saturated aqueous ammonium chloride $(50 \mathrm{~mL})$ and diluted with ethyl acetate $(100 \mathrm{~mL})$. The layers were separated and the aqueous layer was extracted with ethyl acetate $(2 \times 100 \mathrm{~mL})$. The combined organic extracts were dried over anhydrous sodium sulfate, filtered, and concentrated in vacuo. The crude product was then purified by flash chromatography on silica gel to afford $2.5 \mathrm{~g}(74 \%)$ of the title compound as a pale yellow solid, m.p. $82-86{ }^{\circ} \mathrm{C} .{ }^{1} \mathrm{H}$ NMR $\left(500 \mathrm{MHz}, \mathrm{CDCl}_{3}\right) \delta 7.81(\mathrm{~d}, J=9.0 \mathrm{~Hz}, 2 \mathrm{H}), 6.53(\mathrm{~d}, J=9.0 \mathrm{~Hz}, 2$ 
H), 5.79-5.75 (m, $1 \mathrm{H}), 5.71-5.67(\mathrm{~m}, 1 \mathrm{H}), 4.06-4.00(\mathrm{~m}, 1 \mathrm{H}), 3.24-3.16(\mathrm{~m}, 2 \mathrm{H}), 2.81-2.74$ (m, $1 \mathrm{H}), 2.42-2.26(\mathrm{~m}, 2 \mathrm{H}), 2.14-2.06(\mathrm{~m}, 1 \mathrm{H}), 1.78-1.71(\mathrm{~m}, 1 \mathrm{H}), 1.66-1.58(\mathrm{~m}, 1 \mathrm{H}), 1.56$ (s, $9 \mathrm{H}), 1.50-1.42(\mathrm{~m}, 1 \mathrm{H}) ;{ }^{13} \mathrm{C} \mathrm{NMR}\left(125 \mathrm{MHz}, \mathrm{CDCl}_{3}\right) \delta$ 166.1, 151.7, 134.1, 131.3, 131.0, 119.9, 111.1, 79.7, 43.1, 41.9, 35.4, 31.9, 29.7, 28.3; IR (film) 3378, 1682, $1605 \mathrm{~cm}^{-1}$. Anal calcd for $\mathrm{C}_{18} \mathrm{H}_{25} \mathrm{NO}_{2}$ : C, 75.22; $\mathrm{H}, 8.77 ; \mathrm{N}$, 4.87. Found: $\mathrm{C}, 75.09 ; \mathrm{H}, 8.68 ; \mathrm{N}, 4.97$.

$N$-(2-Cyclopent-2-enylethyl)aniline (1e). A flame-dried round-bottom flask was charged with 1,1 '-carbonyl diimidazole $(2.7 \mathrm{~g}, 16.6 \mathrm{mmol})$, purged with argon, and then THF $(20 \mathrm{~mL})$ and 2-cyclopenten-1-acetic acid (2 mL, $2.09 \mathrm{~g}, 16.6 \mathrm{mmol})$ were added via syringe. The mixture was stirred at room temperature for $1 \mathrm{~h}$ and then aniline $(1.5 \mathrm{~mL}, 1.5 \mathrm{~g}, 16.6 \mathrm{mmol})$ was added via syringe and the resulting mixture was stirred at room temperature for $22 \mathrm{~h}$. The reaction mixture was diluted with ethyl acetate $(75 \mathrm{~mL})$ and $\mathrm{H}_{2} \mathrm{O}(50 \mathrm{~mL})$, the layers were separated, and the aqueous layer was extracted with ethyl acetate $(2 \times 100 \mathrm{~mL})$. The combined organic extracts were washed with aqueous $1 \mathrm{M} \mathrm{HCl}(50 \mathrm{~mL})$ and then saturated aqueous $\mathrm{NaHCO}_{3}(50 \mathrm{~mL})$, dried over anhydrous sodium sulfate, filtered, and concentrated in vacuo to afford $3.3 \mathrm{~g}(100 \%)$ of $\mathrm{N}$-phenyl-2-(cyclopent-2-enyl)acetamide ${ }^{6}$ as a tan solid, m.p. $78-80{ }^{\circ} \mathrm{C}\left(\right.$ lit. $\left.85-86^{\circ} \mathrm{C}\right){ }^{6}$

A flame-dried round-bottom flask was charged with $\mathrm{N}$-phenyl-2-(cyclopent-2enyl)acetamide, purged with argon, and then THF $(15 \mathrm{~mL})$ was added via syringe. The resulting solution was cooled to $0{ }^{\circ} \mathrm{C}$ and a solution of $\mathrm{LiAlH}_{4}$ in ether $(50 \mathrm{~mL}, 50 \mathrm{mmol}, 1.0 \mathrm{M})$ was added dropwise via syringe. The reaction mixture was then warmed to room temperature with stirring for $15 \mathrm{~h}$ at which time the starting material had been consumed as judged by TLC analysis. The reaction mixture was cooled to $0{ }^{\circ} \mathrm{C}$, diluted with ether $(300 \mathrm{~mL})$, and quenched slowly with aqueous $10 \mathrm{M} \mathrm{NaOH}$ until all insoluble material had precipitated. The organic supernatant was decanted into a clean Erlenmeyer flask and the precipitate was washed with 
ether $(200 \mathrm{~mL})$. The combined organic extracts were diluted further with hexane $(200 \mathrm{~mL})$, dried over anhydrous sodium sulfate, filtered, and concentrated in vacuo. The crude product was then purified by flash chromatography on silica gel to afford $2.83 \mathrm{~g}(91 \%)$ of the title compound as a colorless oil. ${ }^{1} \mathrm{H}$ NMR (400 MHz, $\mathrm{CDCl}_{3}$ ) $\delta$ 7.25-7.18 (m, $\left.2 \mathrm{H}\right), 6.76-6.71$ (m, $\left.1 \mathrm{H}\right), 6.67-6.62$ (m, $2 \mathrm{H}), 5.83-5.79(\mathrm{~m}, 1 \mathrm{H}), 5.77-5.73(\mathrm{~m}, 1 \mathrm{H}), 3.63(\mathrm{~s}, \mathrm{br}, 1 \mathrm{H}), 3.25-3.14(\mathrm{~m}, 2 \mathrm{H})$, 2.88-2.77 (m, $1 \mathrm{H}), 2.48-2.29$ (m, $2 \mathrm{H}), 2.19-2.08$ (m, $1 \mathrm{H}), 1,83-1.73(\mathrm{~m}, 1 \mathrm{H}), 1.70-1.60$ (m, $1 \mathrm{H}), 1.56-1.46(\mathrm{~m}, 1 \mathrm{H}) ;{ }^{13} \mathrm{C} \mathrm{NMR}\left(100 \mathrm{MHz}, \mathrm{CDCl}_{3}\right) \delta 148.4,134.4,130.8,129.2,117.1$, 112.6, 43.3, 42.5, 35.7, 31.9, 29.8; IR (film) $3410,1603,1506 \mathrm{~cm}^{-1}$. Anal calcd for $\mathrm{C}_{13} \mathrm{H}_{17} \mathrm{~N}: \mathrm{C}$, 83.37; H, 9.15; N, 7.48. Found: C, 83.46; H, 9.17; N, 7.58.

\section{Synthesis of 1,6-diaryloctahydrocyclopenta[b]pyrroles (3a-g)}

General procedure. A flame-dried Schlenk tube was cooled under a stream of argon and charged with $\mathrm{Pd}_{2}(\mathrm{dba})_{3}(1 \mathrm{~mol} \%$ complex, 2 mol \% Pd), 2-(diphenylphosphino)-2'-(N,Ndimethylaminobiphenyl) (11) (2 mol \%), and $\mathrm{NaOt}$-Bu (1.2 equiv). The tube was purged with argon and toluene ( $4 \mathrm{~mL} / \mathrm{mmol}$ amine substrate), the amine substrate (1.0 equiv), and the aryl bromide (1.4 equiv) were added via syringe. The mixture was heated to $110^{\circ} \mathrm{C}$ with stirring until the starting material had been consumed as judged by GC or ${ }^{1} \mathrm{H}$ NMR analysis. The reaction mixture was cooled to room temperature, quenched with saturated aqueous ammonium chloride $(2 \mathrm{~mL})$, and diluted with ethyl acetate $(10 \mathrm{~mL})$. The layers were separated and the aqueous layer was extracted with ethyl acetate $(2 \times 10 \mathrm{~mL})$. The combined organic extracts were dried over anhydrous sodium sulfate, filtered, and concentrated in vacuo. The crude product was then purified by flash chromatography on silica gel. 


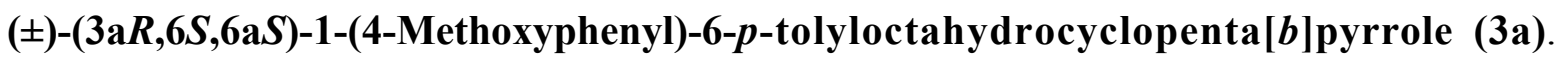
Reaction of $54 \mathrm{mg}(0.25 \mathrm{mmol})$ of $1 \mathrm{a}$ with 4-bromotoluene $(43 \mu \mathrm{L}, 60 \mathrm{mg}, 0.35 \mathrm{mmol})$, and $\mathrm{NaO} t$-Bu (29 mg, $0.3 \mathrm{mmol})$ following the general procedure using dppp as ligand afforded a 25:65:10 mixture of 2:3:4 as the sole detectable products as judged by ${ }^{1} \mathrm{H}$ NMR analysis. This mixture was separated by flash chromatography on silica gel to afford $35 \mathrm{mg}(45 \%)$ of the title compound as a white solid, m.p. 90-92 ${ }^{\circ} \mathrm{C} .{ }^{1} \mathrm{H}$ NMR $\left(400 \mathrm{MHz}, \mathrm{CDCl}_{3}\right) \delta 7.06(\mathrm{~d}, J=8.0 \mathrm{~Hz}, 2$ H), $6.88(\mathrm{~d}, J=8.0 \mathrm{~Hz}, 2 \mathrm{H}), 6.56(\mathrm{~d}, J=9.2 \mathrm{~Hz}, 2 \mathrm{H}), 6.25(\mathrm{~d}, J=9.2 \mathrm{~Hz}, 2 \mathrm{H}), 4.09-4.03(\mathrm{~m}, 1$ H), $3.66(\mathrm{~s}, 3 \mathrm{H}), 3.59-3.52(\mathrm{~m}, 1 \mathrm{H}), 3.14-3.06(\mathrm{~m}, 2 \mathrm{H}), 2.96-2.86(\mathrm{~m}, 1 \mathrm{H}), 2.19$ (s, $3 \mathrm{H})$, 2.19-2.09 (m, $1 \mathrm{H}), 2.04-1.70(\mathrm{~m}, 5 \mathrm{H}) ;{ }^{13} \mathrm{C} \mathrm{NMR}\left(100 \mathrm{MHz}, \mathrm{CDCl}_{3}\right) \delta 150.4,143.3,138.7$, $135.0,129.2,128.1,113.9,113.4,68.1,55.8,51.0,50.9,44.0,33.8,30.9,29.9,20.9$; IR (film) $1511 \mathrm{~cm}^{-1}$. Anal calcd for $\mathrm{C}_{21} \mathrm{H}_{25} \mathrm{NO}: \mathrm{C}, 82.04 ; \mathrm{H}, 8.20 ; \mathrm{N}, 4.56$. Found: $\mathrm{C}, 81.97 ; \mathrm{H}, 8.15 ; \mathrm{N}$, 4.51 .

Use of 11 as ligand for the above transformation afforded a 48:48:2:2 mixture of 2:3:4:5 as judged by ${ }^{1} \mathrm{H}$ NMR analysis of the crude reaction mixture. The desired product was not isolated from this mixture, as superior results were obtained with dppp as ligand (as described above).

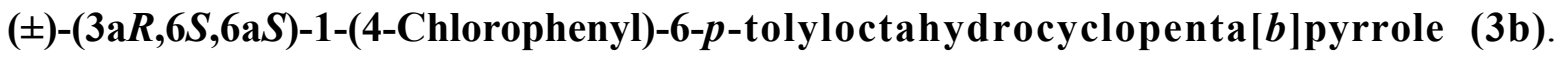
Reaction of $55 \mathrm{mg}(0.25 \mathrm{mmol})$ of $\mathbf{1 b}$ with 4-bromotoluene $(43 \mu \mathrm{L}, 60 \mathrm{mg}, 0.35 \mathrm{mmol})$, and $\mathrm{NaO} t$-Bu $(29 \mathrm{mg}, 0.3 \mathrm{mmol})$ following the general procedure using dppp as ligand afforded a 20:70:10 mixture of 2:3:4 as the sole detectable products as judged by ${ }^{1} \mathrm{H}$ NMR analysis. This mixture was separated by flash chromatography on silica gel to afford $51 \mathrm{mg}(65 \%)$ of the title compound as a white solid, m.p. $107-108{ }^{\circ} \mathrm{C} .{ }^{1} \mathrm{H}$ NMR $\left(500 \mathrm{MHz}, \mathrm{CDCl}_{3}\right) \delta 6.99(\mathrm{~d}, J=8.0 \mathrm{~Hz}$, $2 \mathrm{H}), 6.88-6.82(\mathrm{~m}, 4 \mathrm{H}), 6.18(\mathrm{~d}, J=9.0 \mathrm{~Hz}, 2 \mathrm{H}), 4.13-4.08(\mathrm{~m}, 1 \mathrm{H}), 3.54-3.48(\mathrm{~m}, 1 \mathrm{H})$, 3.17-3.09 (m, $2 \mathrm{H}), 2.96-2.88(\mathrm{~m}, 1 \mathrm{H}), 2.18$ (s, $3 \mathrm{H}), 2.17-2.12(\mathrm{~m}, 1 \mathrm{H}), 2.04-1.98(\mathrm{~m}, 1 \mathrm{H})$, 
$1.96-1.71(\mathrm{~m}, 4 \mathrm{H}) ;{ }^{13} \mathrm{C} \mathrm{NMR}\left(125 \mathrm{MHz}, \mathrm{CDCl}_{3}\right) \delta$ 146.9, 138.3, 135.3, 129.1, 128.2, 127.8, 120.1, 113.5, 67.6, 50.8, 50.3, 44.0, 33.8, 30.7, 29.9, 20.9; IR (film) 1598, $1497 \mathrm{~cm}^{-1}$. Anal calcd for $\mathrm{C}_{20} \mathrm{H}_{22} \mathrm{ClN}$ : C, 77.03; H, 7.11; N, 4.49. Found: $\mathrm{C}, 77.12 ; \mathrm{H}, 7.08 ; \mathrm{N}, 4.62$.

Use of $\mathbf{1 1}$ as ligand for the above transformation afforded a 30:65:2:3 mixture of $\mathbf{2 : 3 : 4 : 5}$ as judged by ${ }^{1} \mathrm{H}$ NMR analysis of the crude reaction mixture. The desired product was not isolated from this mixture, as superior results were obtained with dppp as ligand (as described above).

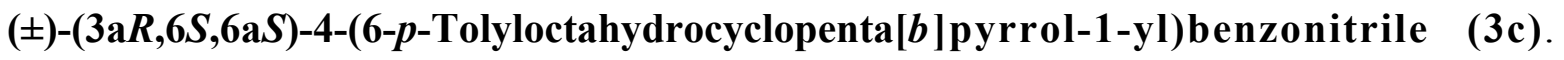
Reaction of $53 \mathrm{mg}(0.25 \mathrm{mmol})$ of $1 \mathrm{c}$ with 4-bromotoluene ( $43 \mu \mathrm{L}, 60 \mathrm{mg}, 0.35 \mathrm{mmol})$, and $\mathrm{NaOt}$ - $\mathrm{Bu}(29 \mathrm{mg}, 0.3 \mathrm{mmol})$ following the general procedure afforded a 10:80:10 mixture of 2:3:4 as the sole detectable products as judged by ${ }^{1} \mathrm{H}$ NMR analysis. This mixture was separated by flash chromatography on silica gel to afford $53 \mathrm{mg}(70 \%)$ of the title compound as a white solid, m.p. $158-160{ }^{\circ} \mathrm{C}$. This material contained ca. $7 \%$ of $( \pm)-(3 \mathrm{a} R, 6 \mathrm{a} S)-4-(1,2,3,3 \mathrm{a}, 4,6 \mathrm{a}-$ hexahydrocyclopenta[b]pyrrol-1-yl)benzonitrile (4c) as an inseparable impurity; data are for the major product. ${ }^{1} \mathrm{H}$ NMR $\left(500 \mathrm{MHz}, \mathrm{CDCl}_{3}\right) \delta 7.12(\mathrm{~d}, J=9.0 \mathrm{~Hz}, 2 \mathrm{H}), 6.93(\mathrm{~d}, J=8.0 \mathrm{~Hz}, 2 \mathrm{H})$, $6.84(\mathrm{~d}, J=8.0 \mathrm{~Hz}, 2 \mathrm{H}), 6.21(\mathrm{~d}, J=9.0 \mathrm{~Hz}, 2 \mathrm{H}), 4.31-4.26(\mathrm{~m}, 1 \mathrm{H}), 3.56-3.51(\mathrm{~m}, 1 \mathrm{H})$, 3.33-3.24 (m, $1 \mathrm{H}), 3.23-3.17(\mathrm{~m}, 1 \mathrm{H}), 3.02-2.94(\mathrm{~m}, 1 \mathrm{H}), 2.25-2.16(\mathrm{~m}, 1 \mathrm{H}), 2.17(\mathrm{~s}, 3 \mathrm{H})$, 2.08-2.02 (m, $1 \mathrm{H}), 1.98-1.81$ (m, $3 \mathrm{H}), 1.80-1.74$ (m, $1 \mathrm{H}) ;{ }^{13} \mathrm{C} \mathrm{NMR}\left(125 \mathrm{MHz}, \mathrm{CDCl}_{3}\right) \delta$ $150.1,137.6,135.8,132.4,129.0,128.3,121.0,112.3,96.4,67.0,50.8,49.8,43.7,33.5,30.5$, 30.1, 20.7; IR (film) 2209, 1604, $1518 \mathrm{~cm}^{-1}$. Anal calcd for $\mathrm{C}_{21} \mathrm{H}_{22} \mathrm{~N}_{2}: \mathrm{C}, 83.40 ; \mathrm{H}, 7.33 ; \mathrm{N}, 9.26$. Found: C, 83.19; H, 7.30; N, 9.29.

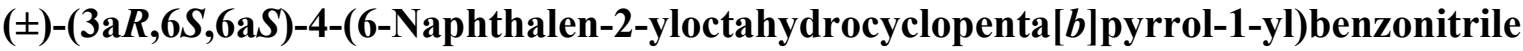

(3d). Reaction of $53 \mathrm{mg}(0.25 \mathrm{mmol})$ of $1 \mathbf{c}$ with 2-bromonaphthalene (72 $\mathrm{mg}, 0.35 \mathrm{mmol})$, and $\mathrm{NaO} t-\mathrm{Bu}(29 \mathrm{mg}, 0.3 \mathrm{mmol})$ following the general procedure afforded $\mathbf{3 d}$ as the sole detectable 
product as judged by ${ }^{1} \mathrm{H}$ NMR analysis. Flash chromatography on silica gel afforded $56 \mathrm{mg}$ (66 \%) of the title compound as a tan solid, m.p. $162-165{ }^{\circ} \mathrm{C} .{ }^{1} \mathrm{H}$ NMR $\left(500 \mathrm{MHz}, \mathrm{CDCl}_{3}\right) \delta 7.68-$ $7.64(\mathrm{~m}, 2 \mathrm{H}), 7.55(\mathrm{~s}, 1 \mathrm{H}), 7.51(\mathrm{~d}, J=8.5 \mathrm{~Hz}, 1 \mathrm{H}), 7.41-7.35(\mathrm{~m}, 2 \mathrm{H}), 7.16(\mathrm{dd}, J=2.0,8.5$ $\mathrm{Hz}, 1 \mathrm{H}), 7.01(\mathrm{~d}, J=8.5 \mathrm{~Hz}, 2 \mathrm{H}), 6.23(\mathrm{~d}, J=8.5 \mathrm{~Hz}, 2 \mathrm{H}), 4.41-4.36(\mathrm{~m}, 1 \mathrm{H}), 3.60-3.54(\mathrm{~m}$, $1 \mathrm{H}), 3.46-3.39$ (m, $1 \mathrm{H}), 3.34-3.27$ (m, $1 \mathrm{H}), 3.07-2.99$ (m, $1 \mathrm{H}), 2.28-2.20$ (m, $1 \mathrm{H}), 2.16-2.04$ (m, $2 \mathrm{H}), 2.01-1.89$ (m, $2 \mathrm{H}), 1.88-1.82(\mathrm{~m}, 1 \mathrm{H}) ;{ }^{13} \mathrm{C} \mathrm{NMR}\left(125 \mathrm{MHz}, \mathrm{CDCl}_{3}\right) \delta$ 150.0, 138.4, 133.0, 132.3, 132.0, 127.8, 127.4, 127.2, 127.2, 126.9, 125.6, 125.3, 120.7, 112.2, 96.6, 67.2, 51.1, 49.8, 43.8, 33.4, 30.6, 30.2; IR (film) $2204,1601,1516 \mathrm{~cm}^{-1}$. Anal calcd for $\mathrm{C}_{24} \mathrm{H}_{22} \mathrm{~N}_{2}$ : C, 85.17; H, 6.55; N, 8.28. Found: C, 84.96; H, 6.41; N, 7.95.

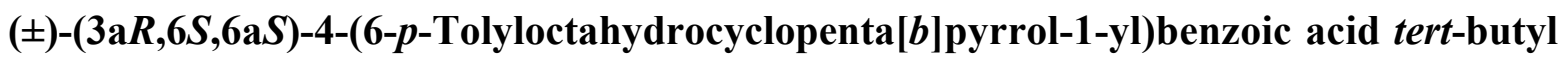
ester (3e). Reaction of $72 \mathrm{mg}(0.25 \mathrm{mmol})$ of $\mathbf{1 d}$ with 4-bromotoluene (43 $\mu \mathrm{L}, 60 \mathrm{mg}, 0.35$ $\mathrm{mmol}$ ), and $\mathrm{NaOt}-\mathrm{Bu}(29 \mathrm{mg}, 0.3 \mathrm{mmol})$ following the general procedure afforded $\mathbf{3 e}$ as the sole detectable product as judged by ${ }^{1} \mathrm{H}$ NMR analysis. Flash chromatography on silica gel afforded $70 \mathrm{mg}(74 \%)$ of the title compound as a white solid, m.p. $100-105{ }^{\circ} \mathrm{C} .{ }^{1} \mathrm{H}$ NMR $(500 \mathrm{MHz}$, $\left.\mathrm{CDCl}_{3}\right) \delta 7.58(\mathrm{~d}, J=8.5 \mathrm{~Hz}, 2 \mathrm{H}), 6.97(\mathrm{~d}, J=8.0 \mathrm{~Hz}, 2 \mathrm{H}), 6.86(\mathrm{~d}, J=7.5 \mathrm{H}, 2 \mathrm{H}), 6.25(\mathrm{~d}, J$ $=9.0 \mathrm{~Hz}, 2 \mathrm{H}), 4.30-4.25(\mathrm{~m}, 1 \mathrm{H}), 3.56-3.51(\mathrm{~m}, 1 \mathrm{H}), 3.32-3.19(\mathrm{~m}, 2 \mathrm{H}), 2.99-2.91(\mathrm{~m}, 1 \mathrm{H})$, 2.22-2.14 (m, $1 \mathrm{H}), 2.16$ (s, $3 \mathrm{H}), 2.09-2.03$ (m, $1 \mathrm{H}), 2.00-1.92$ (m, $1 \mathrm{H}), 1.92-1.80$ (m, $2 \mathrm{H})$, 1.80-1.74 (m, $1 \mathrm{H}), 1.53$ (s, $9 \mathrm{H}) ;{ }^{13} \mathrm{C}$ NMR $\left(125 \mathrm{MHz}, \mathrm{CDCl}_{3}\right) \delta 166.5,150.8,138.0,135.4$, $130.2,129.0,128.3,118.2,111.4,79.4,67.4,50.4,49.9,43.8,33.8,30.5,29.8,28.3,20.9$; IR (film) 1697, 1605, $1518 \mathrm{~cm}^{-1}$. Anal calcd for $\mathrm{C}_{25} \mathrm{H}_{31} \mathrm{NO}_{2}: \mathrm{C}, 79.54 ; \mathrm{H}, 8.28 ; \mathrm{N}, 3.71$. Found: $\mathrm{C}$, $79.57 ; \mathrm{H}, 8.33 ; \mathrm{N}, 3.72$.

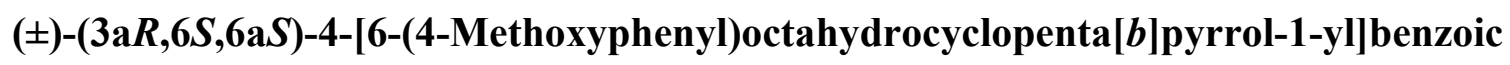
acid tert-butyl ester (3f). Reaction of $72 \mathrm{mg}(0.25 \mathrm{mmol})$ of $\mathbf{1 d}$ with 4-bromoanisole (44 $\mu \mathrm{L}, 65$ 
$\mathrm{mg}, 0.35 \mathrm{mmol})$, and $\mathrm{NaO} t$ - $\mathrm{Bu}(29 \mathrm{mg}, 0.3 \mathrm{mmol})$ following the general procedure afforded a 90:10 mixture of 3:4 as the sole detectable products as judged by ${ }^{1} \mathrm{H}$ NMR analysis. This mixture was separated by flash chromatography on silica gel to afford $59 \mathrm{mg}(60 \%)$ of the title compound as a pale yellow oil. ${ }^{1} \mathrm{H}$ NMR $\left(400 \mathrm{MHz}, \mathrm{CDCl}_{3}\right) \delta 7.58(\mathrm{~d}, J=9.2 \mathrm{~Hz}, 2 \mathrm{H}), 6.99$ (d, $J=8.4 \mathrm{~Hz}, 2 \mathrm{H}), 6.60(\mathrm{~d}, J=8.8 \mathrm{~Hz}, 2 \mathrm{H}), 6.24(\mathrm{~d}, J=8.8 \mathrm{~Hz}, 2 \mathrm{H}), 4.29-4.23(\mathrm{~m}, 1 \mathrm{H}), 3.66$ (s, $3 \mathrm{H}), 3.56-3.50(\mathrm{~m}, 1 \mathrm{H}), 3.32-3.18(\mathrm{~m}, 2 \mathrm{H}), 3.00-2.88(\mathrm{~m}, 1 \mathrm{H}), 2.22-2.13(\mathrm{~m}, 1 \mathrm{H}), 2.09-2.01$ (m, $1 \mathrm{H}), 1.99-1.73(\mathrm{~m}, 4 \mathrm{H}), 1.54(\mathrm{~s}, 9 \mathrm{H}) ;{ }^{13} \mathrm{C} \mathrm{NMR}\left(100 \mathrm{MHz}, \mathrm{CDCl}_{3}\right) \delta 166.4,157.8,150.8$ 133.2, 130.2, 129.9, 118.3, 113.1, 111.4, 79.4, 67.3, 55.1, 49.9, 43.7, 33.7, 30.6, 29.8, 28.3 (two aliphatic resonances are incidentally equivalent); IR (film) 1695, 1605, $1514 \mathrm{~cm}^{-1}$. Anal calcd for $\mathrm{C}_{25} \mathrm{H}_{31} \mathrm{NO}_{3}$ : C, 76.30; H, 7.94; N, 3.56. Found: C, 76.40; H, 7.84; N, 3.57.

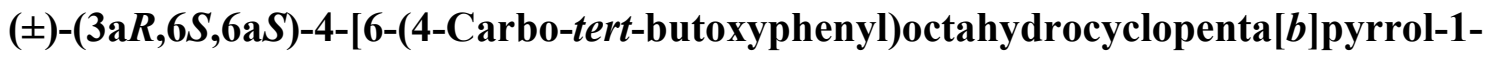
yl]benzoic acid tert-butyl ester (3g). Reaction of $72 \mathrm{mg}(0.25 \mathrm{mmol})$ of 1 d with 4bromobenzoic acid tert-butyl ester $(90 \mathrm{mg}, 0.35 \mathrm{mmol})$, and $\mathrm{NaOt}$-Bu (29 mg, $0.3 \mathrm{mmol})$ following afforded a 30:60:10 mixture of 2:3:4 as the sole products as judged by ${ }^{1} \mathrm{H}$ NMR analysis. This mixture was separated by flash chromatography on silica gel to afford $62 \mathrm{mg}$ (53 \%) of the title compound as a white solid, m.p. $198-200{ }^{\circ} \mathrm{C} .{ }^{1} \mathrm{H} \mathrm{NMR}\left(500 \mathrm{MHz}, \mathrm{CDCl}_{3}\right) \delta 7.68$ $(\mathrm{d}, J=8.5 \mathrm{~Hz}, 2 \mathrm{H}), 7.58(\mathrm{~d}, J=8.5 \mathrm{~Hz}, 2 \mathrm{H}), 7.14(\mathrm{~d}, J=8.5 \mathrm{~Hz}, 2 \mathrm{H}), 6.24(\mathrm{~d}, J=9.0 \mathrm{~Hz}, 2 \mathrm{H})$, 4.31-4.26 (m, $1 \mathrm{H}), 3.59-3.54(\mathrm{~m}, 1 \mathrm{H}), 3.34-3.24(\mathrm{~m}, 2 \mathrm{H}), 3.01-2.92(\mathrm{~m}, 1 \mathrm{H}), 2.24-2.16$ (m, $1 \mathrm{H}), 2.12-2.04(\mathrm{~m}, 1 \mathrm{H}), 2.00-1.76(\mathrm{~m}, 4 \mathrm{H}), 1.53(\mathrm{~s}, 9 \mathrm{H}), 1.52(\mathrm{~s}, 9 \mathrm{H}) ;{ }^{13} \mathrm{C} \mathrm{NMR}(125 \mathrm{MHz}$, $\left.\mathrm{CDCl}_{3}\right) \delta 166.4,165.8,150.6,146.4,130.3,129.6,128.9,128.7,118.7,111.5,80.6,79.6,67.6$ $50.7,50.0,4.0,34.1,30.3,29.7,28.3,28.1$; IR (film) 1700, $1605 \mathrm{~cm}^{-1}$. Anal calcd for $\mathrm{C}_{29} \mathrm{H}_{37} \mathrm{NO}_{4}$ : C, 75.13; H, 8.04; N, 3.02. Found: C, 75.09; H, 7.95; N, 3.03. 


\section{Synthesis of 1,5-diaryloctahydrocyclopenta[b]pyrroles (5a-g)}

General procedure. A flame-dried Schlenk tube was cooled under a stream of argon and charged with $\mathrm{Pd}_{2}(\mathrm{dba})_{3}(1 \mathrm{~mol} \%$ complex, 2 mol \% Pd), di-t-butylmethylphosphonium tetrafluoroborate ( $4 \mathrm{~mol} \%$ ), and $\mathrm{NaO} t-\mathrm{Bu}$ (1.2 equiv). The tube was purged with argon and toluene ( $4 \mathrm{~mL} / \mathrm{mmol}$ amine substrate), the amine substrate (1.0 equiv), and the aryl bromide (1.4 equiv) were added via syringe. The mixture was heated to $110^{\circ} \mathrm{C}$ with stirring until the starting material had been consumed as judged by GC or ${ }^{1} \mathrm{H}$ NMR analysis. The reaction mixture was cooled to room temperature, quenched with saturated aqueous ammonium chloride $(2 \mathrm{~mL})$, and diluted with ethyl acetate $(10 \mathrm{~mL})$. The layers were separated and the aqueous layer was extracted with ethyl acetate $(2 \times 10 \mathrm{~mL})$. The combined organic extracts were dried over anhydrous sodium sulfate, filtered, and concentrated in vacuo. The crude product was then purified by flash chromatography on silica gel.

( \pm )-(3aR,5R,6aR)-1-(4-Methoxyphenyl)-5-p-tolyloctahydrocyclopenta[b]pyrrole (5a). Reaction of $54 \mathrm{mg}(0.25 \mathrm{mmol})$ of $1 \mathrm{a}$ with 4-bromotoluene ( $43 \mu \mathrm{L}, 60 \mathrm{mg}, 0.35 \mathrm{mmol})$, and $\mathrm{NaOt}$ - $\mathrm{Bu}(29 \mathrm{mg}, 0.3 \mathrm{mmol})$ following the general procedure afforded $\mathbf{5 a}$ as the sole detectable product as judged by ${ }^{1} \mathrm{H}$ NMR analysis. Flash chromatography on silica gel afforded $60 \mathrm{mg}$ (78 \%) of the title compound as a pale yellow solid, m.p. $87-89{ }^{\circ} \mathrm{C} .{ }^{1} \mathrm{H} \mathrm{NMR}\left(400 \mathrm{MHz}, \mathrm{CDCl}_{3}\right) \delta$ 7.14-7.07 (m, $4 \mathrm{H}), 6.85$ (d, $J=9.2 \mathrm{~Hz}, 2 \mathrm{H}), 6.63(\mathrm{~d}, J=9.2 \mathrm{~Hz}, 2 \mathrm{H}), 4.21-4.14(\mathrm{~m}, 1 \mathrm{H}), 3.77$ (s, $3 \mathrm{H}), 3.45-3.31(\mathrm{~m}, 2 \mathrm{H}), 3.17-3.05(\mathrm{~m}, 1 \mathrm{H}), 2.95-2.85(\mathrm{~m}, 1 \mathrm{H}), 2.63-2.55$ (m, $1 \mathrm{H}), 2.31$ (s, $3 \mathrm{H}), 2.33-2.25(\mathrm{~m}, 1 \mathrm{H}), 2.18-2.07(\mathrm{~m}, 1 \mathrm{H}), 1.89-1.81(\mathrm{~m}, 1 \mathrm{H}), 1.57-1.41(\mathrm{~m}, 2 \mathrm{H}) ;{ }^{13} \mathrm{C}$ NMR (100 MHz, $\left.\mathrm{CDCl}_{3}\right) \delta 151.0,142.2,140.8,135.5,128.9,126.7,114.8,113.8,64.2,55.9$, 47.8, 45.9, 43.4, 40.8, 40.0, 30.5, 20.9; IR (film) $1513 \mathrm{~cm}^{-1}$. Anal calcd for $\mathrm{C}_{21} \mathrm{H}_{25} \mathrm{NO}$ : C, 82.04; H, 8.20; N, 4.56. Found: C, 81.88; H, 8.22; N, 4.55. 


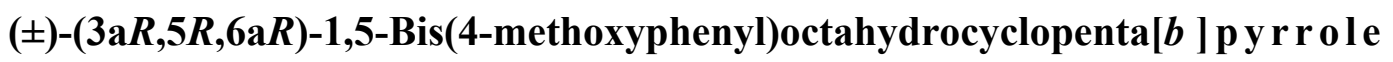

(5b).

Reaction of $54 \mathrm{mg}(0.25 \mathrm{mmol})$ of $1 \mathrm{a}$ with 4-bromoanisole (44 $\mu \mathrm{L}, 65 \mathrm{mg}, 0.35 \mathrm{mmol})$, and $\mathrm{NaO} t$-Bu (29 $\mathrm{mg}, 0.3 \mathrm{mmol})$ following the general procedure afforded $\mathbf{5 b}$ as the sole detectable product as judged by ${ }^{1} \mathrm{H}$ NMR analysis. Flash chromatography on silica gel afforded $62 \mathrm{mg}(77$ \%) of the title compound as a pale yellow oil. ${ }^{1} \mathrm{H} \mathrm{NMR}\left(400 \mathrm{MHz}, \mathrm{CDCl}_{3}\right) \delta 7.14(\mathrm{~d}, J=8.4 \mathrm{~Hz}$, $2 \mathrm{H}), 6.87-6.80(\mathrm{~m}, 4 \mathrm{H}), 6.62(\mathrm{~d}, J=8.8 \mathrm{~Hz}, 2 \mathrm{H}), 4.21-4.14(\mathrm{~m}, 1 \mathrm{H}), 3.78(\mathrm{~s}, 3 \mathrm{H}), 3.77(\mathrm{~s}, 3$ H), 3.44-3.31 (m, 2 H), 3.15-3.04 (m, $1 \mathrm{H}), 2.94-2.84(\mathrm{~m}, 1 \mathrm{H}), 2.62-2.54(\mathrm{~m}, 1 \mathrm{H}), 2.32-2.23$ (m, $1 \mathrm{H}), 2.17-2.07(\mathrm{~m}, 1 \mathrm{H}), 1.88-1.80(\mathrm{~m}, 1 \mathrm{H}), 1.54-1.39(\mathrm{~m}, 2 \mathrm{H}) ;{ }^{13} \mathrm{C} \mathrm{NMR}(100 \mathrm{MHz}$, $\left.\mathrm{CDCl}_{3}\right) \delta 157.8,150.9,142.2,136.0,127.7,114.8,113.8,113.6,64.2,55.9,55.2,47.8,45.4$ 43.3, 40.9, 40.1, 30.5; IR (film) $1512 \mathrm{~cm}^{-1}$. Anal calcd for $\mathrm{C}_{21} \mathrm{H}_{25} \mathrm{NO}_{2}: \mathrm{C}, 77.98 ; \mathrm{H}, 7.79 ; \mathrm{N}$, 4.33. Found: C, 78.05; H, 7.87; N, 4.36.

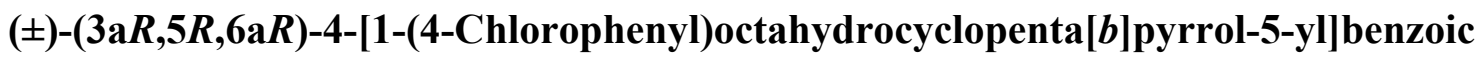

acid tert-butyl ester (5c). Reaction of $55 \mathrm{mg}(0.25 \mathrm{mmol})$ of $\mathbf{1 b}$ with 4-bromobenzoic acid tertbutyl ester (90 mg, $0.35 \mathrm{mmol})$, and $\mathrm{NaOt}-\mathrm{Bu}(29 \mathrm{mg}, 0.3 \mathrm{mmol})$ following the general procedure afforded a 15:85 mixture of 3:5 as the sole detectable products as judged by ${ }^{1} \mathrm{H}$ NMR analysis. This mixture was separated by flash chromatography on silica gel to afford $70 \mathrm{mg}$ (71 $\%$ ) of the title compound as a white solid, m.p. $136-138{ }^{\circ} \mathrm{C} .{ }^{1} \mathrm{H}$ NMR $\left(500 \mathrm{MHz}, \mathrm{CDCl}_{3}\right) \delta 7.88$ $(\mathrm{d}, J=8.5 \mathrm{~Hz}, 2 \mathrm{H}), 7.23(\mathrm{~d}, J=8.5 \mathrm{~Hz}, 2 \mathrm{H}), 7.15(\mathrm{~d}, J=9.0 \mathrm{~Hz}, 2 \mathrm{H}), 6.53(\mathrm{~d}, J=9.0 \mathrm{~Hz}, 2 \mathrm{H})$, 4.22-4.16 (m, $1 \mathrm{H}), 3.45-3.36(\mathrm{~m}, 2 \mathrm{H}), 3.24-3.15$ (m, $1 \mathrm{H}), 2.97-2.88$ (m, $1 \mathrm{H}), 2.68-2.61$ (m, $1 \mathrm{H}), 2.33-2.26(\mathrm{~m}, 1 \mathrm{H}), 2.16-2.08(\mathrm{~m}, 1 \mathrm{H}), 1.91-1.84(\mathrm{~m}, 1 \mathrm{H}), 1.58(\mathrm{~s}, 9 \mathrm{H}), 1.59-1.52(\mathrm{~m}, 1$ $\mathrm{H}), 1.51-1.42(\mathrm{~m}, 1 \mathrm{H}) ;{ }^{13} \mathrm{C} \mathrm{NMR}\left(125 \mathrm{MHz}, \mathrm{CDCl}_{3}\right) \delta 165.7,148.5,145.5,130.0,129.5,128.8$, 126.7, 120.6, 113.7, 80.7, 63.6, 47.3, 46.2, 43.6, 40.5, 39.3, 30.0, 28.2; IR (film) 1709, 1600, 
$1499 \mathrm{~cm}^{-1}$. Anal calcd for $\mathrm{C}_{24} \mathrm{H}_{28} \mathrm{ClNO}_{2}: \mathrm{C}, 72.44 ; \mathrm{H}, 7.09 ; \mathrm{N}, 3.52$. Found: $\mathrm{C}, 72.59 ; \mathrm{H}, 7.17 ; \mathrm{N}$, 3.58 .

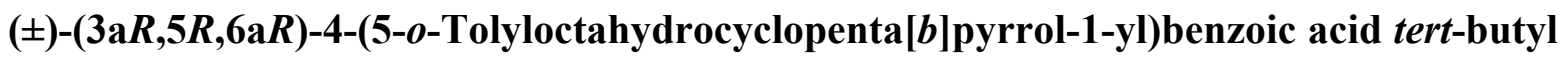
ester (5d). Reaction of $72 \mathrm{mg}(0.25 \mathrm{mmol})$ of $\mathbf{1 d}$ with 2-bromotoluene (42 $\mu \mathrm{L}, 60 \mathrm{mg}, 0.35$ $\mathrm{mmol})$, and $\mathrm{NaOt}$-Bu $(29 \mathrm{mg}, 0.3 \mathrm{mmol})$ following the general procedure afforded a 10:90 mixture of 3:5 as the sole detectable products as judged by ${ }^{1} \mathrm{H}$ NMR analysis. This mixture was separated by flash chromatography on silica gel to afford $66 \mathrm{mg}(70 \%)$ of the title compound as a white solid, m.p. $46-48{ }^{\circ} \mathrm{C} .{ }^{1} \mathrm{H}$ NMR $\left(500 \mathrm{MHz}, \mathrm{CDCl}_{3}\right) \delta 7.86(\mathrm{~d}, J=9.0 \mathrm{~Hz}, 2 \mathrm{H}), 7.22-7.18$ (m, $1 \mathrm{H}), 7.16-7.07(\mathrm{~m}, 3 \mathrm{H}), 6.57(\mathrm{~d}, J=9.0 \mathrm{~Hz}, 2 \mathrm{H}), 4.31-4.25(\mathrm{~m}, 1 \mathrm{H}), 3.56-3.51(\mathrm{~m}, 2 \mathrm{H})$, 3.39-3.30 (m, $1 \mathrm{H}), 2.99-2.90(\mathrm{~m}, 1 \mathrm{H}), 2.70-2.63$ (m, $1 \mathrm{H}), 2.38$ (s, $3 \mathrm{H}), 2.26-2.20$ (m, $1 \mathrm{H})$, 2.18-2.09 (m, $1 \mathrm{H}), 1.96-1.90$ (m, $1 \mathrm{H}), 1.63-1.43$ (m, $2 \mathrm{H}), 1.58$ (s, $9 \mathrm{H}) ;{ }^{13} \mathrm{C}$ NMR $(125 \mathrm{MHz}$, $\left.\mathrm{CDCl}_{3}\right) \delta 166.4,149.8,141.2,135.7,131.0,130.2,126.1,126.0,125.0,118.5,111.5,79.6,63.3$ 46.9, 43.6, 42.2, 40.1, 38.2, 29.6, 28.3, 19.7; IR (film) 1695, 1605, $1521 \mathrm{~cm}^{-1}$. Anal calcd for $\mathrm{C}_{25} \mathrm{H}_{31} \mathrm{NO}_{2}$ : C, 79.54; H, 8.28; N, 3.71. Found: C, 79.44; H, 8.20; N, 3.73.

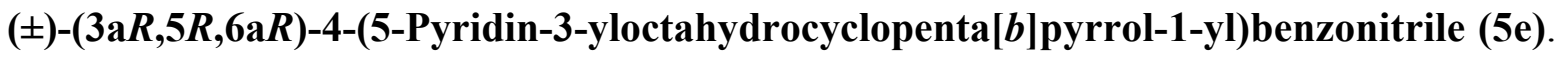
Reaction of $53 \mathrm{mg}(0.25 \mathrm{mmol})$ of 1c with 3-bromopyridine (34 $\mu \mathrm{L}, 55 \mathrm{mg}, 0.35 \mathrm{mmol})$, and $\mathrm{NaO} t$-Bu (29 mg, $0.3 \mathrm{mmol})$ following the general procedure afforded a 5:10:10:75 mixture of 2:3:4:5 as judged by ${ }^{1} \mathrm{H}$ NMR analysis. This mixture was separated by flash chromatography on silica gel to afford $50 \mathrm{mg}(69 \%)$ of the title compound as a yellow oil. This material contained ca. $7 \%$ of $( \pm)-(3 \mathrm{a} R, 6 S, 6 \mathrm{a} S)-4-(6-$ pyridin-3-yloctahydrocyclopenta[b]pyrrol-1-yl)benzonitrile (3) as an inseparable impurity; data are for the major product. ${ }^{1} \mathrm{H}$ NMR $\left(400 \mathrm{MHz}, \mathrm{CDCl}_{3}\right) \delta$ 8.46-8.44 (m, $1 \mathrm{H}), 8.42(\mathrm{dd}, J=1.2,4.8 \mathrm{~Hz}, 1 \mathrm{H}), 7.50-7.45(\mathrm{~m}, 1 \mathrm{H}), 7.42(\mathrm{~d}, J=8.8 \mathrm{~Hz}, 2 \mathrm{H})$, $7.18(\mathrm{dd}, J=4.8,8.0 \mathrm{~Hz}, 1 \mathrm{H}), 6.54(\mathrm{~d}, J=8.8 \mathrm{~Hz}, 2 \mathrm{H}), 4.29-4.21(\mathrm{~m}, 1 \mathrm{H}), 3.53-3.45(\mathrm{~m}, 2 \mathrm{H})$, 
3.24-3.14 (m, $1 \mathrm{H}), 3.00-2.94(\mathrm{~m}, 1 \mathrm{H}), 2.78-2.68(\mathrm{~m}, 1 \mathrm{H}), 2.36-2.27(\mathrm{~m}, 1 \mathrm{H}), 2.19-2.08(\mathrm{~m}$, $1 \mathrm{H}), 1.96-1.89$ (m, $1 \mathrm{H}), 1.60-1.50$ (m, $1 \mathrm{H}), 1.49-1.39$ (m, $1 \mathrm{H}) ;{ }^{13} \mathrm{C} \mathrm{NMR}\left(400 \mathrm{MHz}, \mathrm{CDCl}_{3}\right.$ ) ठ 149.2, 148.8, 147.8, 138.4, 133.9, 133.3, 123.2, 120.6, 112.3, 97.3, 63.3, 46.9, 43.7, 43.5, 40.6, 38.6, 29.4; IR (film) 2210, 1605, $1520 \mathrm{~cm}^{-1}$. Anal calcd for $\mathrm{C}_{19} \mathrm{H}_{19} \mathrm{~N}_{3}: \mathrm{C}, 78.86 ; \mathrm{H}, 6.62 ; \mathrm{N}$, 14.52. Found: C, 78.28; H, 6.61; N, 14.37 .

\section{$( \pm)-(3 \mathrm{a} R, 5 R, 6 \mathrm{a} R)-$ Phenyl[4-(1-phenyloctahydrocyclopenta[b]pyrrol-5-}

yl)phenyl]methanone (5f). Reaction of $47 \mathrm{mg}(0.25 \mathrm{mmol})$ of $1 \mathbf{e}$ with 4-bromobenzophenone (91 $\mathrm{mg}, 0.35 \mathrm{mmol})$, and $\mathrm{NaOt}-\mathrm{Bu}(29 \mathrm{mg}, 0.3 \mathrm{mmol})$ following the general procedure afforded a 10:90 mixture of 3:5 as the sole detectable products as judged by ${ }^{1} \mathrm{H}$ NMR analysis. This mixture was separated by flash chromatography on silica gel to afford $80 \mathrm{mg}(87 \%)$ of the title compound as a yellow oil. ${ }^{1} \mathrm{H} \mathrm{NMR}\left(400 \mathrm{MHz}, \mathrm{CDCl}_{3}\right) \delta 7.79(\mathrm{~d}, J=7.2 \mathrm{~Hz}, 2 \mathrm{H}), 7.73$ (d, $J=$ $8.4 \mathrm{~Hz}, 2 \mathrm{H}), 7.61-7.56(\mathrm{~m}, 1 \mathrm{H}), 7.51-7.45$ (m, $2 \mathrm{H}), 7.33$ (d, J = 8.0 Hz, $2 \mathrm{H}), 7.28-7.22$ (m, 2 H), 6.73-6.64 (m, 3 H), 4.30-4.23 (m, 1 H), 3.53-3.42 (m, 2 H), 3.31-3.20 (m, 1 H), 3.01-2.90 (m, $1 \mathrm{H}), 2.77-2.69(\mathrm{~m}, 1 \mathrm{H}), 2.39-2.31(\mathrm{~m}, 1 \mathrm{H}), 2.20-2.09(\mathrm{~m}, 1 \mathrm{H}), 1.94-1.86(\mathrm{~m}, 1 \mathrm{H})$, $1.68-1.52(\mathrm{~m}, 2 \mathrm{H}) ;{ }^{13} \mathrm{C} \mathrm{NMR}\left(400 \mathrm{MHz}, \mathrm{CDCl}_{3}\right) \delta$ 196.2, 149.0, 147.0, 137.9, 135.5, 132.1, 130.2, 129.8, 129.0, 128.1, 126.8, 115.9, 112.7, 63.6, 47.1, 46.2, 43.6, 40.8, 39.3, 30.1; IR (film) $1655,1598,1504 \mathrm{~cm}^{-1}$. Anal calcd for $\mathrm{C}_{26} \mathrm{H}_{25} \mathrm{NO}$ : C, 84.98; H, 6.86; N, 3.81. Found: C, 84.63; $\mathrm{H}, 6.95 ; \mathrm{N}, 3.81$.

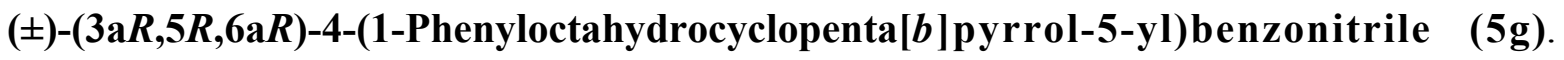
Reaction of $47 \mathrm{mg}$ ( $0.25 \mathrm{mmol})$ of $1 \mathbf{e}$ with 4-bromobenzonitrile $(64 \mathrm{mg}, 0.35 \mathrm{mmol})$, and $\mathrm{NaOt}$ $\mathrm{Bu}(29 \mathrm{mg}, 0.3 \mathrm{mmol})$ following the general procedure afforded $\mathbf{5 g}$ as the sole detectable product as judged by ${ }^{1} \mathrm{H}$ NMR analysis. Flash chromatography on silica gel afforded $56 \mathrm{mg}(78 \%)$ of the title compound as a white solid, m.p. 80-82 ${ }^{\circ} \mathrm{C} .{ }^{1} \mathrm{H}$ NMR $\left(500 \mathrm{MHz}, \mathrm{CDCl}_{3}\right) \delta 7.55(\mathrm{~d}, J=8.5$ 
$\mathrm{Hz}, 2 \mathrm{H}), 7.31$ (d, $J=8.0 \mathrm{~Hz}, 2 \mathrm{H}), 7.27-7.22(\mathrm{~m}, 2 \mathrm{H}), 6.73-6.69(\mathrm{~m}, 1 \mathrm{H}), 6.65(\mathrm{~d}, J=8.0 \mathrm{~Hz}$ 2 H), 4.29-4.23 (m, $1 \mathrm{H}), 3.48-3.44$ (m, $2 \mathrm{H}), 3.27-3.18$ (m, $1 \mathrm{H}), 3.00-2.91$ (m, $1 \mathrm{H}), 2.74-2.66$ (m, $1 \mathrm{H}), 2.35-2.28(\mathrm{~m}, 1 \mathrm{H}), 2.18-2.10(\mathrm{~m}, 1 \mathrm{H}), 1.92-1.86(\mathrm{~m}, 1 \mathrm{H}), 1.59-1.47(\mathrm{~m}, 2 \mathrm{H}) ;{ }^{13} \mathrm{C}$ NMR (125 MHz, $\left.\mathrm{CDCl}_{3}\right) \delta 149.5,146.8,132.1,129.1,127.7,119.0,115.9,112.7,109.8,63.4$, 47.0, 46.2, 43.6, 40.6, 39.1, 29.9; IR (film) 2226, 1598, $1505 \mathrm{~cm}^{-1}$. Anal calcd for $\mathrm{C}_{20} \mathrm{H}_{20} \mathrm{~N}_{2}$ : C, 83.30; H, 6.99; N, 9.71. Found: C, 83.25; H, 7.10; N, 9.82.

\section{Oxidative Cyclization of (2-Cyclopent-2-enylethyl)-(4-methoxyphenyl)amine (1a)}

( \pm )-(3aR,6aS)-1-(4-Methoxyphenyl)-1,2,3,3a,4,6a-hexahydrocyclopenta[b]pyrrole (4). ${ }^{2} \quad \mathrm{~A}$ flame-dried Schlenk tube was cooled under a stream of argon and charged with $\operatorname{Pd}_{2}\left(\mathrm{dba}_{3}\right)_{3}(2.3$ $\mathrm{mg}, 0.0025 \mathrm{mmol}, 1 \mathrm{~mol} \%$ ), ligand (0.01 mmol, $4 \mathrm{~mol} \%$ ), and $\mathrm{NaO} t-\mathrm{Bu}(29 \mathrm{mg}, 0.3 \mathrm{mmol})$. The tube was purged with argon and toluene $(1 \mathrm{~mL}), \mathbf{1 a}(54 \mathrm{mg}, 0.25 \mathrm{mmol})$, and 4bromotoluene ( $43 \mu \mathrm{L}, 60 \mathrm{mg}, 0.35 \mathrm{mmol}$ ) were added via syringe. The mixture was heated to $110{ }^{\circ} \mathrm{C}$ with stirring until the starting material had been consumed as judged by $\mathrm{GC}$ analysis. The reaction mixture was cooled to room temperature, quenched with saturated aqueous ammonium chloride $(2 \mathrm{~mL})$, and diluted with ethyl acetate $(10 \mathrm{~mL})$. The layers were separated and the aqueous layer was extracted with ethyl acetate $(2 \times 10 \mathrm{~mL})$. The combined organic extracts were dried over anhydrous sodium sulfate, filtered, and concentrated in vacuo. The crude product was then purified by flash chromatography on silica gel to afford the title compound as a colorless oil. The yields obtained with four different ligands are shown below in Table S1. ${ }^{1} \mathrm{H}$ NMR (500 $\left.\mathrm{MHz}, \mathrm{CDCl}_{3}\right) \delta 6.86(\mathrm{~d}, J=9.5 \mathrm{~Hz}, 2 \mathrm{H}), 6.65(\mathrm{~d}, J=9.0 \mathrm{~Hz}, 2 \mathrm{H}), 5.94-5.92(\mathrm{~m}, 1 \mathrm{H})$, 5.81-5.79 (m, $1 \mathrm{H}), 4.58-4.55(\mathrm{~m}, 1 \mathrm{H}), 3.77(\mathrm{~s}, 3 \mathrm{H}), 3.34-3.29(\mathrm{~m}, 1 \mathrm{H}), 3.14-3.08(\mathrm{~m}, 1 \mathrm{H})$, 2.99-2.86 (m, $1 \mathrm{H}), 2.64-2.58$ (m, $1 \mathrm{H}), 2.24-2.18$ (m, $2 \mathrm{H}), 1.74-1.67$ (m, $1 \mathrm{H})$. 
Table S1: Synthesis of 4

\begin{tabular}{|c|c|}
\hline Ligand & Isolated Yield \\
\hline $\mathrm{P}(2 \text {-furyl })_{3}$ & $53 \%$ \\
\hline $\mathrm{PMePh}_{2}$ & $44 \%$ \\
\hline $\mathrm{PMe}_{2} \mathrm{Ph}$ & $54 \%$ \\
\hline $\mathrm{PMe}_{3}$ & $61 \%$ \\
\hline
\end{tabular}

\section{$N$-Arylation of $N$-(2-cyclopent-2-enylethyl)-p-anisidine (1a)}

$N$-(2-Cyclopent-2-enylethyl)- $N$-(4-methylphenyl)-p-anisidine (2). A flame-dried Schlenk tube was cooled under a stream of argon and charged with $\mathrm{Pd}_{2}(\mathrm{dba})_{3}(2.3 \mathrm{mg}, 0.0025 \mathrm{mmol}, 1$ mol \%), 2-(di-tert-butylphosphino)biphenyl (10) (1.8 mg, $0.005 \mathrm{mmol}, 2 \mathrm{~mol} \%$ ), and $\mathrm{NaO} t-\mathrm{Bu}$ (29 $\mathrm{mg}, 0.3 \mathrm{mmol})$. The tube was purged with argon and toluene $(1 \mathrm{~mL}), \mathbf{1 a}(54 \mathrm{mg}, 0.25 \mathrm{mmol})$, and 4-bromotoluene ( $37 \mu \mathrm{L}, 51 \mathrm{mg}, 0.3 \mathrm{mmol}$ ) were added via syringe. The resulting mixture was heated to $60^{\circ} \mathrm{C}$ with stirring until the starting material had been consumed as judged by GC analysis. The reaction mixture was cooled to room temperature, quenched with saturated aqueous ammonium chloride $(2 \mathrm{~mL})$, and diluted with ethyl acetate $(10 \mathrm{~mL})$. The layers were separated and the aqueous layer was extracted with ethyl acetate $(2 \times 10 \mathrm{~mL})$. The combined organic extracts were dried over anhydrous sodium sulfate, filtered, and concentrated in vacuo. The crude product was then purified by flash chromatography on silica gel to afford $71 \mathrm{mg}$ (92 \%) of the title compound as a colorless oil. ${ }^{1} \mathrm{H}$ NMR $\left(400 \mathrm{MHz}, \mathrm{CDCl}_{3}\right) \delta$ 7.05-7.00 (m, $\left.4 \mathrm{H}\right)$, $6.88(\mathrm{~d}, J=8.6 \mathrm{~Hz}, 2 \mathrm{H}), 6.73(\mathrm{~d}, J=8.6 \mathrm{~Hz}, 2 \mathrm{H}), 5.77-5.73(\mathrm{~m}, 1 \mathrm{H}), 5.71-5.67(\mathrm{~m}, 1 \mathrm{H}), 3.82$ (s, 3 H), 3.70-3.62 (m, 2 H), 2.77-2.66 (m, 1 H), 2.42-2.24 (m, 2 H), 2.28 (s, 3 H), 2.13-2.04 (m, $1 \mathrm{H}), 1.84-1.74(\mathrm{~m}, 1 \mathrm{H}), 1.69-1.59(\mathrm{~m}, 1 \mathrm{H}), 1.50-1.40(\mathrm{~m}, 1 \mathrm{H}) ;{ }^{13} \mathrm{C}$ NMR (100 MHz, 
$\left.\mathrm{CDCl}_{3}\right) \delta 155.6,146.6,141.4,134.5,130.6,129.5,127.9,125.6,117.2,114.7,55.5,51.1,43.3$, 33.5, 31.9, 29.8, 20.4; IR (film) 1616, $1508 \mathrm{~cm}^{-1}$. Anal calcd for $\mathrm{C}_{21} \mathrm{H}_{25} \mathrm{NO}$ : C, 82.04; $\mathrm{H}, 8.20$; N, 4.56. Found: C, 81.97; H, 8.15; N, 4.46.

\section{Assignment of Stereochemistry}

\section{1,6-Diaryloctahydrocyclopenta[b]pyrroles (3a-g)}

The stereochemistry of ( \pm )-(3 a $R, 6 S, 6 \mathrm{a} S)$-1-(4-Methoxyphenyl)-6- $p$ tolyloctahydrocyclopenta[b]pyrrole (3a) was assigned on the basis of nOe signals as shown below.

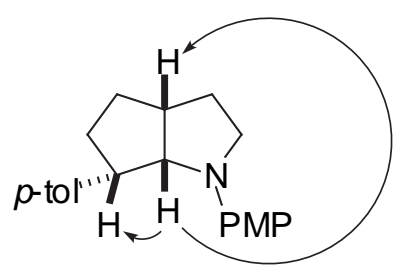

3a

The stereochemistry of the 1,6-diaryloctahydrocyclopenta[b]pyrrole products $\mathbf{3 b}-\mathbf{g}$ was assigned based on analogy to the above example.

\section{1,5-Diaryloctahydrocyclopenta[b]pyrroles (5a-g)}

The stereochemistry of $( \pm)-(3 \mathrm{a} R, 5 R, 6 \mathrm{a} R)-1-(4-M e t h o x y p h e n y l)-5-p-$ tolyloctahydrocyclopenta[b]pyrrole (5a) was assigned on the basis of nOe signals as shown below.

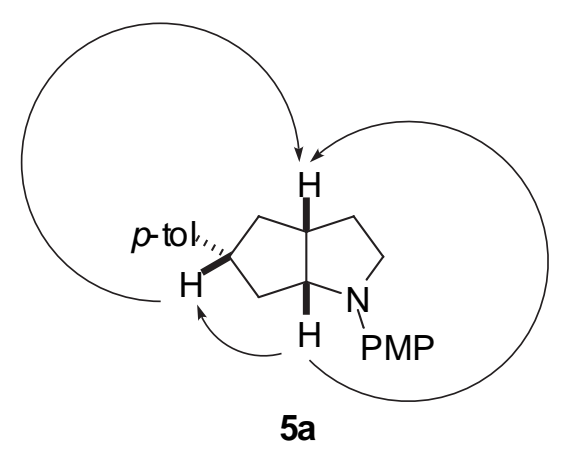


The stereochemistry of the 1,5-diaryloctahydrocyclopenta[b]pyrrole products $\mathbf{5 b} \mathbf{b}-\mathbf{g}$ was assigned based on analogy to the above example.

\section{Ligand Effects ${ }^{a}$}

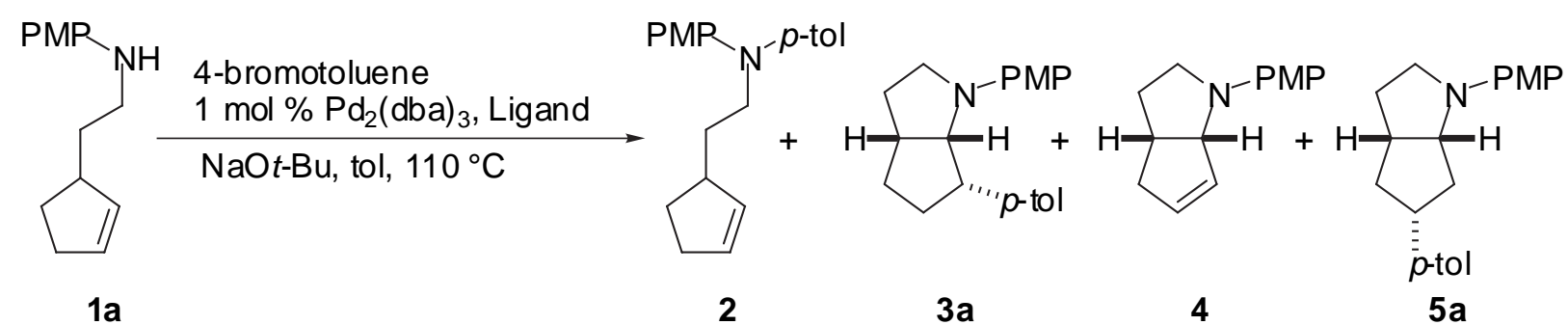

\begin{tabular}{|c|c|c|c|c|c|}
\hline Entry & Ligand & 2 & $3 \mathbf{a}$ & 4 & $5 \mathbf{a}$ \\
\hline 1 & $\mathrm{Pd}\left[\mathrm{P}(t-\mathrm{Bu})_{3}\right]_{2}$ & 100 & 0 & 0 & 0 \\
\hline $2^{b}$ & $\mathrm{P}(t-\mathrm{Bu})_{3} \cdot \mathrm{HBF}_{4}$ & 98 & 2 & 0 & 0 \\
\hline 3 & $t$-Bu ${ }_{2} \mathrm{P}(o$-biphenyl) (10) & 94 & 6 & 0 & 0 \\
\hline 4 & Dpp-benzene & 87 & 3 & 7 & 4 \\
\hline 5 & $\mathrm{Pd}\left[\mathrm{P}(t-\mathrm{Bu})_{2} \mathrm{Cy}\right]_{2}$ & 81 & 5 & 0 & 14 \\
\hline 6 & $\begin{array}{l}\text { 2-(Dicyclohexylphosphino)-2'- } \\
\text { (N,N-dimethylamino)biphenyl }\end{array}$ & 76 & 24 & 0 & 0 \\
\hline 7 & $\begin{array}{l}\text { 2-(Diphenylphosphino)-2'-(N,N- } \\
\text { dimethylamino)biphenyl (11) }\end{array}$ & 56 & 40 & 2 & 2 \\
\hline 8 & Xantphos & 54 & 46 & 0 & 0 \\
\hline 9 & $\mathrm{Cy}_{2} \mathrm{P}(o$-biphenyl $)$ & 52 & 39 & 1 & 8 \\
\hline 10 & dppp & 31 & 58 & 8 & 3 \\
\hline 11 & dppf & 27 & 42 & 12 & 19 \\
\hline 12 & $\mathrm{P}(o-\text { tol })_{3}$ & 18 & 42 & 9 & 31 \\
\hline $13^{b, c}$ & $\mathrm{PMe}_{3} \cdot \mathrm{HBF}_{4}$ & 0 & 0 & 98 & 2 \\
\hline $14^{b, \mathrm{c}}$ & $\mathrm{PEt}_{3} \cdot \mathrm{HBF}_{4}$ & 0 & 0 & 92 & 8 \\
\hline
\end{tabular}




\begin{tabular}{|c|c|c|c|c|c|}
\hline $15^{b}$ & $\mathrm{PMe}_{2} \mathrm{Ph}$ & 0 & 0 & 92 & 8 \\
\hline 16 & $\mathrm{PMePh}_{2}$ & 0 & 0 & 86 & 14 \\
\hline 17 & $\mathrm{P}(2 \text {-furyl })_{3}$ & 0 & 8 & 82 & 10 \\
\hline 18 & dppe-p-OMe & 3 & 4 & 69 & 24 \\
\hline $19^{d}$ & dppe & 5 & 7 & 63 & 25 \\
\hline 20 & $\mathrm{P}\left[\mathrm{C}_{6} \mathrm{H}_{4}(p-\mathrm{OMe})\right]_{3}$ & 0 & 5 & 59 & 36 \\
\hline $21^{d}$ & Dppb & 4 & 10 & 56 & 30 \\
\hline 22 & $\mathrm{PPh}_{3}$ & 3 & 9 & 55 & 33 \\
\hline 23 & Dppm & 12 & 11 & 51 & 26 \\
\hline 24 & $( \pm)$-BINAP & 32 & 9 & 37 & 22 \\
\hline 25 & Dpe-phos & 19 & 27 & 37 & 17 \\
\hline 26 & dppe- $p-\mathrm{CF}_{3}$ & 20 & 25 & 28 & 27 \\
\hline 27 & $\mathrm{MeP}(t-\mathrm{Bu})_{2} \bullet \mathrm{HBF}_{4}$ & 0 & 0 & 0 & 100 \\
\hline 28 & $\operatorname{Pd}\left[\mathrm{MeP}(t-\mathrm{Bu})_{2}\right]_{2}$ & 0 & 0 & 0 & 100 \\
\hline 29 & $\mathrm{Pd}\left[\mathrm{PCy}_{3}\right]_{2}$ & 1 & 2 & 1 & 96 \\
\hline 30 & $\mathrm{PCy}_{3}$ & 2 & 2 & 6 & 90 \\
\hline 31 & $\mathrm{Pd}\left[\mathrm{P}(t-\mathrm{Bu}) \mathrm{Cy}_{2}\right]_{2}$ & 6 & 6 & 0 & 88 \\
\hline 32 & Dppf-i-Pr & 13 & 12 & 0 & 75 \\
\hline 33 & dcpe & 11 & 4 & 19 & 66 \\
\hline 34 & $\mathrm{P}\left[\mathrm{C}_{6} \mathrm{H}_{4}\left(p-\mathrm{CF}_{3}\right)\right]_{3}$ & 3 & 28 & 26 & 43 \\
\hline 35 & $\mathrm{P}\left[\mathrm{C}_{6} \mathrm{H}_{4}\left(m-\mathrm{CF}_{3}\right)\right]_{3}$ & 4 & 31 & 32 & 33 \\
\hline 36 & $\mathrm{P}\left[\mathrm{C}_{6} \mathrm{H}_{4}\left(o-\mathrm{CF}_{3}\right)\right]_{3}$ & \multicolumn{4}{|c|}{ Very Low Conversion } \\
\hline 37 & $\mathrm{P}\left(\mathrm{C}_{6} \mathrm{~F}_{5}\right)_{3}$ & \multicolumn{4}{|c|}{ Very Low Conversion } \\
\hline 38 & $\begin{array}{l}\text { Tris(2,4,6-trimethoxyphenyl) } \\
\text { phosphine }\end{array}$ & \multicolumn{4}{|c|}{ Very Low Conversion } \\
\hline
\end{tabular}




\begin{tabular}{|c|l|c|}
\hline 39 & Trimesitylphosphine & Complex Mixture \\
\hline
\end{tabular}

(a) Conditions: 1.0 equiv 1a, 2.0 equiv 4-bromotoluene, 1.2 equiv $\mathrm{NaO} t \mathrm{Bu}, 1 \mathrm{~mol} \% \mathrm{Pd}_{2}\left(\mathrm{dba}_{3}\right.$, $2 \mathrm{~mol} \%$ ligand (bis phosphines) or $4 \mathrm{~mol} \%$ ligand (mono phosphines), toluene $(0.25 \mathrm{M}), 110{ }^{\circ} \mathrm{C}$. The product ratios refer to GC ratios of products 2-5 that have been corrected for GC response factor. All reactions proceed to completion unless otherwise noted. (b) The reaction was conducted with 1.4 equiv 4-bromotoluene. (c) The reaction proceeded only to c.a. $80 \%$ conversion. (d) Approximately 3\% of an imine side product derived from substrate oxidation was detected by GC and GC/MS analysis of the crude reaction mixture.

\footnotetext{
${ }^{1}$ Lee, J. -s.; Velarde-Ortiz, R.; Guijarro, A.; Wurst, J. R.; Rieke, R. D. J. Org. Chem. 2000, 65, $5428-5430$.

${ }^{2}$ Ney, J. E.; Wolfe, J. P. Angew. Chem. Int. Ed. 2004, 43, 3605-3608.

${ }^{3}$ Chatt, J.; Hussain, W.; Leigh, G. J.; Ali, H. M.; Pickett, C. J.; Rankin, D. A. J. Chem. Soc., Dalton Trans. 1985, 1131-1136.

${ }^{4}$ Eapen, K. C.; Tamborski, C. J. Fluorine Chem. 1980, 15, 239-243.

${ }^{5}$ Friedrichsen, B. P.; Powell, D. R.; Whitlock, H. W. J. Am. Chem. Soc. 1990, 112, 8931-8941.

${ }^{6}$ Nicolaou, K. C.; Baran, P. S.; Zhong, Y. -L.; Sugita, K. J. Am. Chem. Soc. 2002, 124, 2212-2220.
} 